\title{
Threshold-based Energy Efficient Clustering Protocol for Corrosion Risk Monitoring of Reinforced Concrete
}

\author{
Vijayalakshmi Senniappan \\ Associate Professor \\ Department of \\ Information Technology \\ Park College of Engineering \\ and Technology
}

\author{
Jayashree Subramanian \\ Professor \\ Department of Computer \\ Science and Engineering \\ PSG College of Technology
}

\begin{abstract}
In recent years, wireless sensor networks (WSN) are being used by a wide range of applications. Sensor nodes are used for gathering data about the various physical parameters of the system under study. As sensor nodes are battery powered, energy efficiency is an important challenge faced by WSN. Clustering is a technique for improving the energy efficiency of the sensor network. The reported piece of work uses the soft computing technique, Biogeography-Based Krill Herd Algorithm (BBKH) for energy efficient clustering that results in improving the network lifetime. While cluster heads are selected, the rate adjustment is made for congestion mitigation. Likewise, a threshold based data forwarding method is also employed for further improvement of the network lifetime. Based on threshold principle, sending of similarly sensed data repeatedly is avoided. The network lifetime is compared before and later on using the threshold value. The obtained results after using the threshold concept are $18.80 \%$ better than its threshold free counterpart. The proposed method is used in structural health monitoring (SHM) application where the corrosion risk is monitored in the reinforced concrete structures.
\end{abstract}

\section{Keywords}

Structural Health Monitoring, Congestion, BiogeographyBased Krill Herd algorithm, Genetic Algorithm, Clustering, Corrosion

\section{INTRODUCTION}

Structural Health Monitoring is a procedure employed for monitoring the physical status of civil structures. Buildings are prone to several types of damages. Timely warning of the stipulation of the building helps to initiate rehabilitation at the proper instant. Lack of timely maintenance leads to loss of human life and belongings. WSN helps to detect damages occurring in building constructions. Sensor Nodes (SN) are used for measuring different parameters which cause damages to civil structures. As $\mathrm{SN}$ is battery powered, the energy efficiency of these nodes is a common issue in WSN, which affects the network performance. In this research work, energy efficient clusters are formed to assist in the improvement of network lifetime. Clustering is a technique to group SN deployed in different locations. Each cluster will take in a Cluster Head $(\mathrm{CH})$. $\mathrm{CH}$ collects the sensed data from the $\mathrm{SN}$ and aggregates them and transmits to the Base Station (BS) for further processing. Energy is consumed for sensing the data, transmitting, receiving and aggregating the data. While the data are transmitted to the $\mathrm{CH}$, congestion occurs. Congestion prevails when the buffer present in the $\mathrm{CH}$ overflows due to more number of incoming data, followed by packet drops. The dropped packets are to be retransmitted, which further drops the energy of the nodes. Hence, congestion is to be avoided. Also, if all the sensed data which are similar, are transmitted to $\mathrm{CH}$, the energy consumed would be more. Instead, a threshold sensitive data forwarding method can be employed to send data only when the value is more capital than a threshold value, by avoiding the transmission of all the similar sensed data.

Every year lots of structural collapses occur around the world. The Federal Highway Administration (FHWA) has surveyed in 1991 that $39 \%$ of highway structure defects are due to reinforcement corrosion. The ASCE 2009 Report for America's Infrastructure shows that USD \$ 2,2 trillion is necessary for the next 5 years to bring America's structures up to the working conditions. The U.S.A. Federal Highway administration stated in 2011 that $11 \%$ of the national bridges are structurally defective $[15,16]$. This can result in fatal accidents such as the collapse of the I-35W Mississippi River Bridge on the 1st of August 2007 [17]. Due to these accidents, not only causes human casualties, but has also economic impact due to extra travel cost. Hence, regular monitoring of damages is essential for drawing out the life of the constructions.

The objective of the proposed method is to avoid damage and save human life and property. Motivated by this idea, the proposed method has been applied in the application area of SHM to monitor the corrosion risk in the reinforced concrete. Once corrosion occurs, it leads to loss of reinforcement section, loss of steel-concrete bond, concrete cracking, spalling, etc., which reduces the service life of the structures. Corrosion sensors are used for sensing five factors, namely, linear polarization resistance, open circuit potential, resistivity, chloride ion concentration and temperature [20]. Corrosion sensors deployed in different positions of the buildings will monitor the values periodically. If there is no corrosion, the data sensed periodically will be alike. If all the sensed data are broadcast to the $\mathrm{CH}$, more energy will be expended. Alternatively, if the sensed values are more than a threshold value, it can be transmitted to $\mathrm{CH}$ for monitoring the corrosion risk of the reinforced concrete. The proposed method uses a soft computing technique, namely, Biogeography-Based Krill Herd algorithm (BBKH) for improving the network lifetime by selecting optimal $\mathrm{CH}$, while considering congestion mitigation for data collection and selective forwarding of corrosion data based on their threshold values.

\section{RELATED WORK}

A great deal of research work has been carried out in the past for making energy efficient clusters for improving the network lifetime. The first well-known clustering protocol 
developed is Low Energy Adaptive Clustering hierarchy with Deterministic CH selection (LEACH) [2]. Using this method the $\mathrm{CH}$ are selected with optimal probability. In each round, different $\mathrm{CH}$ are selected for data collection. Energy Efficient Two Levels Distributed Clustering (EE-TLDC) [3] has proposed a scheme in which the number of $\mathrm{CHs}$ is limited to reduce the transmission cost. In this, the primary layer of $\mathrm{CH}$ is selected with probability and the low levels of $\mathrm{CH}$ are selected from these primary $\mathrm{CH}$ based on the distance from BS.

Many deeds have been borne out in the literature using soft computing techniques for clustering of nodes. A Genetic Algorithm (GA) based approach is proposed in [4] for $\mathrm{CH}$ selection. A Particle Swarm Optimization (PSO) [5] algorithm is utilized to choose an optimal set of $\mathrm{CH}$ in each cycle. The $\mathrm{CH}$ are selected by combining PSO with LEACH. A Genetic Algorithm based Energy - Efficient Adaptive clustering Protocol (GAEEP) [6] was proposed. The GAEEP runs for many rounds, with each round having two phases, namely setup phase and steady state phase. During the setup phase the clusters are formed and in the next phase, the sensed data are transferred to $\mathrm{CH}$. The results shown by GAEEP are better compared with LEACH.

A Threshold Sensitive Advanced Distributed Energy Efficient Clustering Routing Protocol for Wireless Sensor Networks (TADEEC) [7] was developed to improve the network communication. Not entirely the data sensed by the network are channeled to the $\mathrm{CH}$. Instead, a node transmits when the currently sensed data is more capital than a hard threshold value. A Threshold sensitive Energy Efficient sensor network protocol (TEEN) [8] was used for a temperature sensing application. In this protocol, the nodes react immediately to any unusual or drastic change coming on the sensing value. The protocol suits well for time critical applications, where immediate decisions have to be arrived at based on the sensed value.

\section{THEORETICAL BACKGROUND}

\subsection{Corrosion process}

The primary causes of corrosion occurrence in steel support are due to carbonation and chloride attack. A protective oxide film is present on the airfoil of the steel reinforcement, due to high alkalinity of concrete. Carbonation occurs when the protective layer will be lost by reaction of $\mathrm{CO}_{2}$ present in the environment with the alkaline. The presence of chloride ions may also break the passive film of steel. Chlorides may penetrate into the concrete through various sources like sea water, diffusion of an environment, harmful salts coming from cement, aggregates, water and mixtures, concrete cover, temperature and relative humidity [1]. The chloride penetration can be assessed by Fiber Optic Sensors (FOS). In [9], FOS has been used for monitoring the chloride penetration in a bridge spanning $67 \mathrm{~m}$ long. In [10-14], FOS has been used for chloride limit monitoring. Figure 1 shows corrosion in steel due to chloride attack [33].

Corrosion of reinforcement occurs within a few years from the fourth dimension of the building. Repairing of corrosion damage is very unmanageable, as it requires a number of steps, namely, the removal of carbonated concrete, cleaning of reinforcement, application of protective coat, making getting the reduced blade area, applying bond on the coat and cover replacement. Both carbonation and chloride attack drastically increase the corrosion process in the reinforcing steel $[18,19]$.

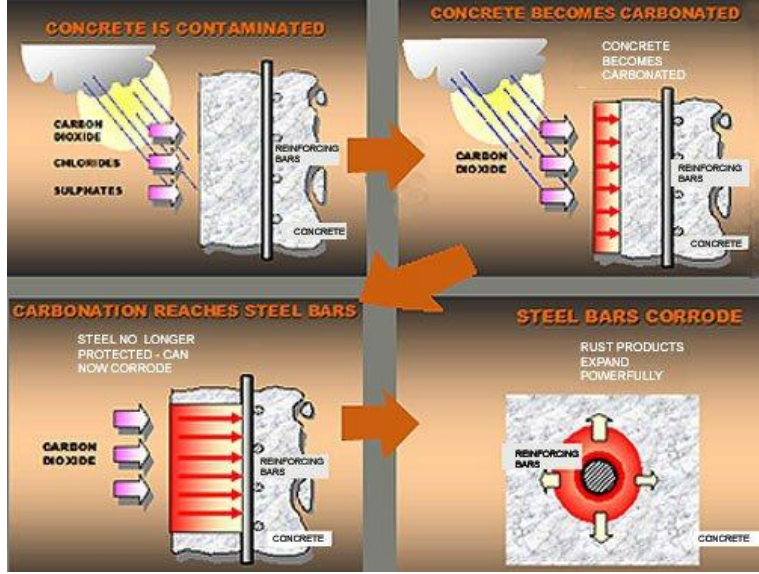

Fig 1: Chloride attack in steel reinforcement

Two cases of corrosion exist in the steel reinforcement: normal corrosion and pitting corrosion. In normal corrosion, a passive layer of corrosion is formed on the surface stratum of the steel, which reduces further corrosion [18]. Whereas, pitting corrosion occurs when the passive film is interrupted due to increase in chloride ion increase. Pitting corrosion increases very rapidly compared to normal corrosion leading to crack formation in the concrete structures. Deterioration takes place in two stages due to corrosion: initiation and propagation stage. In the initiation phase, no significant deterioration is noticed, whereas in propagation stage more deterioration is seen and corrosion rate is more.

\subsection{Corrosion Monitoring Sensor}

Several corrosion sensors are available for monitoring the corrosion in concrete, namely Fiber Optic Sensor (FOS) [9], Embedded Corrosion Instrument (ECI) [20], Embedded Capacity Sensor [21] etc. The ECI sensors are mostly used in construction and maintenance of civil structures [22]. Fig.2 shows an ECI sensor [22].

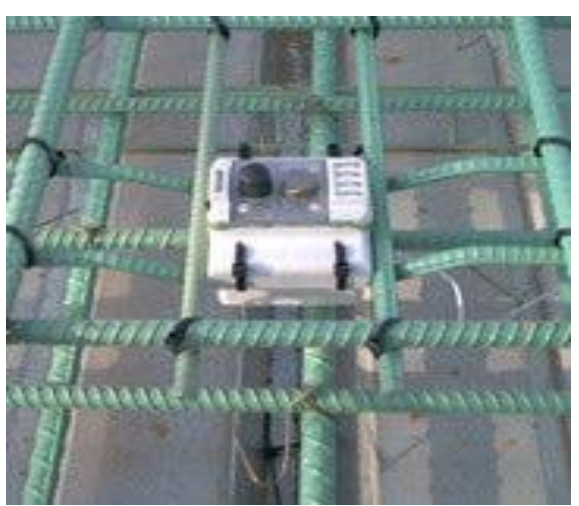

Fig 2: ECI sensor

A corrosion sensor has macrocells consisting of black steel (anode) and a noble metal (cathode). Several anodes are placed in concrete at defined cover depths. The cathodes are positioned near the anodes. Different measurement types exist for corrosion measurement. Some of the measurements are electrical current, electrochemical potential, temperature and electrolytic resistance of concrete [20]. The proposed method considers the electrochemical potential measurement for determining the corrosion risk assessment. The potential is a measure of the tendency of any metal's reaction to the environment. In reinforced concrete structure, the reaction between the reinforcement and the concrete develops a potential, which is assessed by the corrosion sensor. The 
common method of detection of corrosion is the half-cell potential (HCP) measurements. Monitoring the open circuit potential (OCP) is a procedure for review of concrete constructions. A reduction of the electrochemical potential is an indication of the onset of corrosion. The potential value is measured with respect to a reference electrode, such as saturated calomel electrode (SCE), copper/copper sulfate electrode (CSE), silver/silver chloride electrode etc. The risk of corrosion related to HCP, as per ASTM C 876 [23], is given in Table.1. The OCP method mostly used for corrosion monitoring as proposed by [31].

Table 1. Corrosion risk related to HCP measurement

\begin{tabular}{|l|l|l|l|}
\hline \multicolumn{2}{|l|}{$\begin{array}{l}\text { Open Circuit potential (OCP) values in } \\
\text { millivoltmeter (mV) }\end{array}$} & $\begin{array}{l}\text { Corrosion } \\
\text { condition }\end{array}$ \\
\cline { 1 - 3 } $\begin{array}{l}\text { Copper/copper } \\
\text { sulfate }\end{array}$ & $\begin{array}{l}\text { Silver/silver } \\
\text { chloride }\end{array}$ & Calomel & $\begin{array}{l}\text { Low (10\% risk } \\
\text { of corrosion) }\end{array}$ \\
\hline$>-200 \mathrm{mV}$ & $>-106 \mathrm{mV}$ & $>-126 \mathrm{mv}$ & $\begin{array}{l}\text { Intermediate } \\
\text { corrosion risk }\end{array}$ \\
\hline-200 to & -106 to -256 & -126 to \\
$-350 \mathrm{mV}$ & $\mathrm{mV}$ & $276 \mathrm{mV}$ & $\begin{array}{l}\text { High (<90\% } \\
\text { risk of } \\
\text { corrosion) }\end{array}$ \\
\hline$<-350 \mathrm{mV}$ & $<-256 \mathrm{mV}$ & $<276 \mathrm{mV}$ & $\begin{array}{l}\text { Severe } \\
\text { corrosion }\end{array}$ \\
\hline$<-500 \mathrm{mV}$ & $<-406 \mathrm{mV}$ & $\mathrm{V}$ & $-426 \mathrm{~m}$ \\
\hline
\end{tabular}

According to [32], the negative values of the potential is because in a half-cell system, usually the positive terminal is connected to the steel and the negative terminal is connected to the half-cell sensor. Half-cell can measure negative potential values. The negative sign of the potential value depends on how the leads are connected between the half-cell and the rebar. If the OCP values go beyond the ranges as given in Table 1, the civil structure is under the risk of corrosion. Early maintenance prevents further deterioration of the construction. OCP values in Table 1 provide the corrosion risk probability, merely not the rate of corrosion. With the help of the potential range, the corrosion risk of the reinforced concrete structure can be understood.

\section{BBKH BASED CH SELECTION}

Krill Herd $(\mathrm{KH})$ [24] is a search algorithm developed in 2012. KH comes under a meta-heuristic algorithm, inspired by the bio-based swarm intelligence algorithm. The KH models the herding behavior of Krill swarms. The objective function is defined based on the density of krill individuals and the distance from the food. $\mathrm{KH}$ has the advantage of owning a few control variables to adjust in comparison with other algorithms of similar form. The location of a krill individual is struck by three actions $[25,26]$ :

(i) Movement affected by other krill $\left(\mathrm{N}_{\mathrm{i}}\right)$

(ii) Foraging action $\left(\mathrm{F}_{\mathrm{i}}\right)$

(iii) Random diffusion $\left(\mathrm{D}_{\mathrm{i}}\right)$

The objective function is measured at each iteration after the above three actions, to discover the best krill and its location.
The fitness function is calculated as given in Eqn.1 as per Lagrangian model [24].

$$
\frac{d x_{i}}{d t}=N_{i}+F_{i}+D_{i}
$$

The movement brought out is given by Eqn (2).

$$
N_{i}^{\text {new }}=N^{\text {max }} \propto_{i}+\omega_{n} N_{i}^{\text {old }}
$$

Where $\alpha_{i}$ is the direction, $N_{\max }$ is the maximum speed, $\omega_{\mathrm{n}}$ represents the inertia weight and $\mathrm{N}_{\mathrm{i}}^{\text {old }}$ is the last motion induced.The foraging action of an individual is calculated as given in Eqn (3).

$$
F_{i}=V_{f} \beta_{i}+\omega_{f} F_{i}^{o l d}
$$

where

$$
\beta_{i}=\beta_{i}{ }^{\text {food }}+\beta_{i}^{\text {best }}
$$

In Eqn (3), $V_{f}$ represents the foraging speed, $\omega_{f}$ gives the inertia weight and $F_{i}^{\text {old }}$ is the last foraging motion value. $\beta_{i}^{\text {food }}$ represents the effect due to the presence of food and $\beta_{i}{ }^{\text {best }}$ is the effect due to the current krill's best fitness value recorded. The random diffusion helps for the hearding mechanism of the krill as given in Eqn (5).

$$
D_{i}=D^{\max } \delta
$$

$\mathrm{D}_{\max }$ gives the maximum diffusion speed and $\delta$ is the random directional vector with values between $[-1,1]$.The new position of $\mathrm{KH}$ at $t+\Delta t$ is found using as per the Eqn. (6).

$$
X_{i}(t+\Delta t)=X_{i}(t)+\Delta t \frac{d X_{i}}{d t}
$$

$\Delta \mathrm{t}$ represents the scale factor. The optimal result is given by $\mathrm{KH}$ by exploring the search space effectively, but not constantly, as KH may be stuck in local optima. To overcome this drawback of $\mathrm{KH}$, a krill migration (KM) operator is used, to exploit the search space fully and apply the best global optimum solution. The usage of KM operator was motivated by a meta-heuristic evolution algorithm given in [27]. BBKH algorithm is a combination of $\mathrm{KH}$ and $\mathrm{KM}$ operator.

\section{FITNESS CALCULATION}

Corrosion sensors are deployed in locations where the onset of corrosion is more expected. Sensor nodes collect corrosion values and transmit them to cluster head. The cluster head is selected using BBKH algorithm. The algorithm runs through many cycles until the terminating condition of a fixed number rounds is reached. In each round, a multiobjective fitness function is assessed. The fitness function used is given in Equation (7).

$$
F=w 1 *\left(\frac{1}{F I}\right)+w 2 * \sum_{i}^{n} B O
$$

where

$$
\begin{aligned}
& \text { FI }- \text { Fairness Index } \\
& \text { BO - Buffer Occupancy } \\
& \text { w1, w } 2 \text { - weight, w1 } 1=0.7 \text {, w } 2=0.3
\end{aligned}
$$

Based on the performance metrics discussion given by [28], Fairness Index (FI) is defined as in Equation (8).

$F I=\frac{\left(\Sigma_{f} T_{f}\right)^{2}}{\eta \Sigma_{\mathrm{f}}\left(\mathrm{T}_{\mathrm{f}}\right)^{2}}$

where $T_{f}$ is the throughput of flow $f, \eta$ is the number of flows. The fitness function determines the best $\mathrm{CH}$ in each cycle by employing BBKH algorithm. The Fairness Index and buffer 
occupancy level are considered while $\mathrm{CH}$ is selected. The throughput is kept fairly among all the flows coming to a $\mathrm{CH}$ by FI. FI is to be maximized. To mitigate congestion, the buffer occupancy level $\mathrm{BO}$ is minimized. The congestion occurrence is mitigated by considering the buffer occupancy level. The buffer is used for storing the incoming packets. If the incoming data rate is more, packets overflow in the buffer. Hence, if the incoming rate is reduced, the packet overflow is avoided. The BBKH algorithm selects a rate for data transfer of the incoming packets based on the present buffer occupancy level of the $\mathrm{CH}$. The rate is selected such that the buffer level are kept lower. Based on the rate selection given by $\mathrm{BBKH}$, the data are transmitted from sensor nodes to $\mathrm{CH}$, so that buffer overflow is mitigated. As no packet drops prevail, no retransmission will be there. Hence, energy consumption is less.

To further improve the energy efficiency of the sensor nodes, selective transmission of the sensed data to $\mathrm{CH}$ takes place. The non-clustered nodes under each $\mathrm{CH}$ sense the data periodically. If there is no corrosion, the data sensed by the sensors during different time intervals may be same. It is unnecessary to send the similar data measured repeatedly to the $\mathrm{CH}$, as data transmission and reception consume energy. Hence, in the suggested method, two threshold parameters, namely, hard and soft threshold [29,30] are employed while the data are forwarded to $\mathrm{CH}$. An SN transmits the data sensed only when the current sensed value is more outstanding than the hard threshold and the conflict between current sensed value and the previously sensed data is more outstanding than the soft threshold. The soft threshold is a little change in the sensed value. For example, the hard threshold for corrosion monitoring is considered as -106 for the Silver/Silver electrode used as given in Table.1 Once when the sensed value is equal to -106 , it is transmitted to the $\mathrm{CH}$. This value is stored in the node as a sensed value (SV). After the predefined time interval, the data is sensed again. Let the current sensed data be represented as a CV. If the CV value is greater than $\mathrm{SV}$ and the minor conflict is seen between $\mathrm{SV}$ and $\mathrm{CV}$, then the $\mathrm{CV}$ value is broadcast to the $\mathrm{CH}$ and the old $\mathrm{SV}$ is replaced with $\mathrm{CV}$ value. Hence, the onset of the corrosion is identified, only when the threshold value is arrived at. This serves the civil engineers for rehabilitation of the damaged building structure. The potential value for different electrodes is listed in Table.1.

\section{SIMULATION ENVIRONMENT}

The work is simulated using MATLAB. The parameters considered during the simulation are listed as in Table 2. The energy model of the proposed work is similar to the energy model of LEACH [2].

Table 2 Simulation parameters

\begin{tabular}{|l|l|}
\hline Parameter & Value \\
\hline Number of nodes & 100 \\
\hline Dimension of Sensing region & $200 * 200 \mathrm{~m}$ \\
\hline $\mathrm{E}_{\text {elec }}$ & $50 \mathrm{~nJ} / \mathrm{bit}$ \\
\hline $\mathrm{E}_{\mathrm{DA}}$ & $5 \mathrm{~nJ} / \mathrm{bit}$ \\
\hline$\varepsilon_{\mathrm{fs}}$ & $10 \mathrm{pJ} /\left(\mathrm{bit}_{\mathrm{m}}{ }^{2}\right)$ \\
\hline$\varepsilon_{\mathrm{mp}}$ & $0.0013 \mathrm{pJ} /\left(\mathrm{bit}_{\mathrm{m}} \mathrm{m}^{4}\right)$ \\
\hline
\end{tabular}

The necessary number of sensor nodes is deployed in positions which are prone to hurt. The algorithm BBKH runs for 1500 rounds. Each round selects $\mathrm{CH}$ based on the fitness value and clusters formed. Each sensor node periodically senses the data, compares with the hard and soft threshold values and then if a deviation is there it sends the data to the $\mathrm{CH}$. The BBKH algorithm is compared with the classical algorithm GA. In each round, several parameters are calculated for evaluating the performance of BBKH. The algorithm is run until the stopping condition is achieved.

\section{RESULTS AND DISCUSSION}

The outcomes are compared for different performance aspects considered. The results demonstrate the comparison of $\mathrm{BBKH}$ with GA. Initially, the algorithm is simulated without considering thresholding concept ie. all the data sensed about the corrosion are transmitted to the $\mathrm{CH}$. Fig. 3 (a) to (e) gives the results obtained by BBKH and GA without applying thresholding concept.

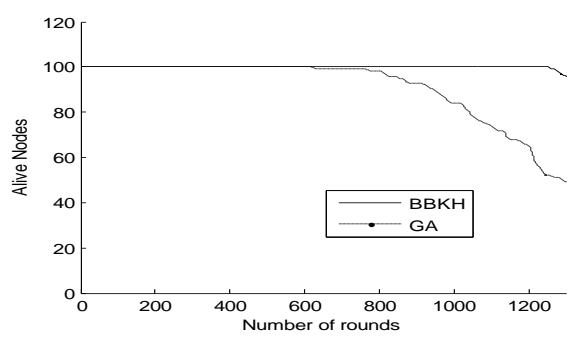

Fig 3 (a): Number of Alive nodes (without thresholding)

In Fig.3 (a), the number of alive nodes is compared. It can be observed that the performance of BBKH in comparison with GA is better. Equally, if the buffer occupancy level is within lower limits, no packet drop occurs. This avoids packet retransmission. Hence, energy consumption is less leading to the prolonged life of the sensor nodes.

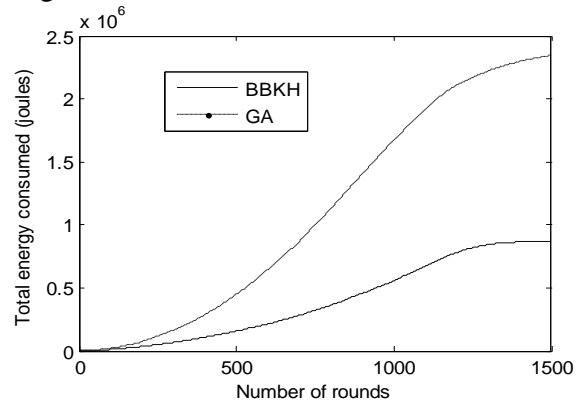

Fig. 3 (b) : Total energy consumed (without thresholding)

By comparing the total energy consumption, it is apparent that the BBKH performs better than the GA as shown in fig.3(b). In fig. 3(a), the number of alive nodes is more, indicating that the energy consumption is less. If energy consumption is more, then dead nodes would occur in earlier rounds. In GA, energy usage is more comparable with BBKH.

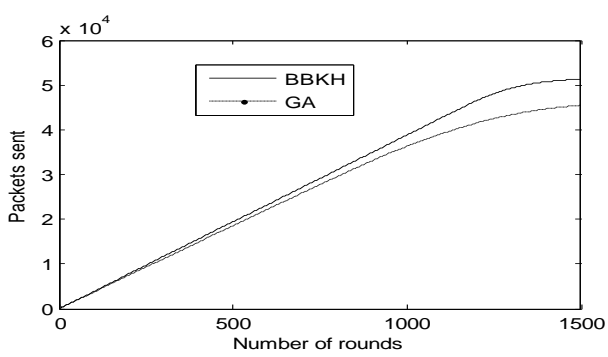

Fig 3 (c) : Total packets transmitted (without thresholding) 
In fig 3(c), the total packets transmitted by the different algorithms are compared. If congestion is mitigated by considering the buffer occupancy level and by also considering the fairness index for $\mathrm{CH}$ selected, less packet drop occurs. Most of the received packets are transmitted. Also, the limited occupancy level of packets in the buffer avoids the packet drop, which helps in the transmission of all the incoming packets. The packets sent by BBKH show better outcomes in comparison to GA.

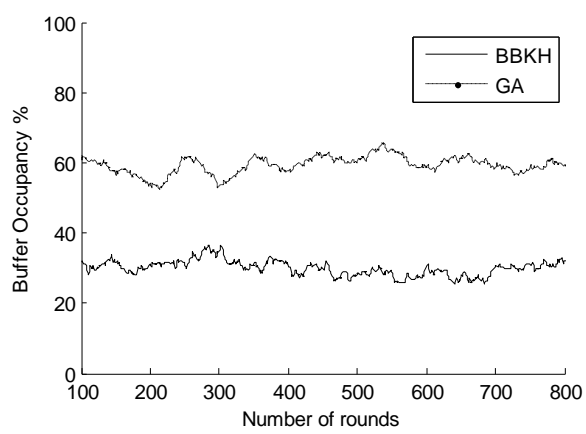

Fig 3 (d): Buffer occupancy (without thresholding)

By considering the buffer occupancy position of the GA, BBKH shows good results as it is seen in fig 3(d). BBKH maintains lower limits of buffer occupancy to avoid packet drop due to congestion. In the GA, the buffer occupancy is more in comparison with BBKH, which leads to increased packet drops. Retransmission takes place, by taking in more energy. This is evident from the previous results.

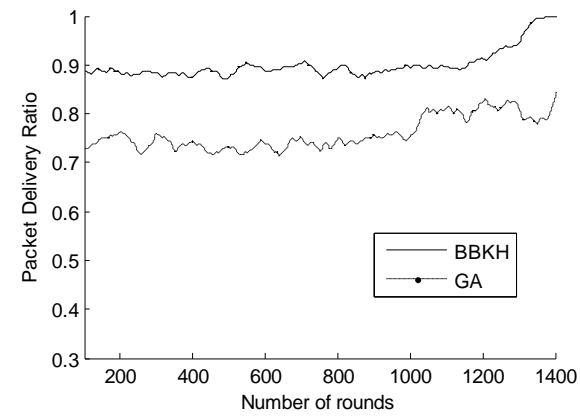

Fig 3(e) : Packet Delivery ratio (without thresholding)

In the end, the comparability of the packet delivery ratio (PDR) shows better outcomes for the proposed BBKH algorithm as shown in fig 3(e). High PDR is attributed to lower buffer occupancy, with less packet drop. Low PDR, infers that the packet is retransmitted, by increasing the energy uptake and reducing the network lifetime.

The algorithm was later simulated by considering thresholding concept i.e sensed data about the corrosion are selectively transmitted to the $\mathrm{CH}$ based on their threshold limits. The solutions have improved by nearly $18.80 \%$ when comparing the previous results which do not consider threshold factor. In the proposed work, BBKH outperforms in all the network parameters considered. Therefore, the lifetime of the sensors deployed in the building is prolonged for extended years.

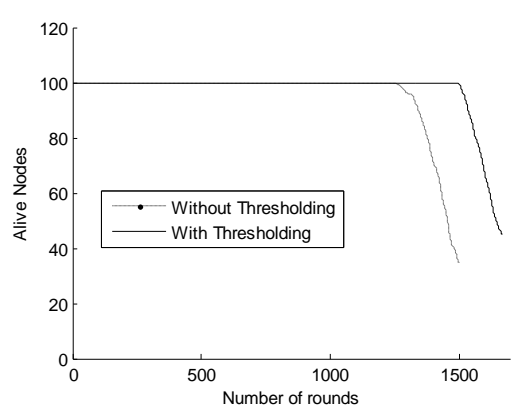

Fig 4 (a): Number of alive nodes by using BBKH

Fig. 4 (a) to (e) gives the performance of BBKH with and without threshold concept. The number of alive nodes as shown in Fig.4. (a), has improved after applying threshold value as only selected data are sent to $\mathrm{CH}$. Hence, the lifespan of the nodes has improved considerably.

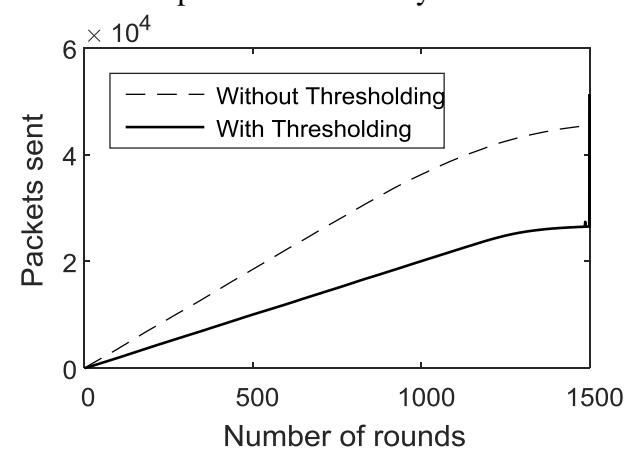

Fig 4 (b) : Total packets sent by using BBKH

From Fig 4 (b), it is evident that the total number of packets is reduced after thresholding, as the proposed method sends only limited information based on the threshold limits.

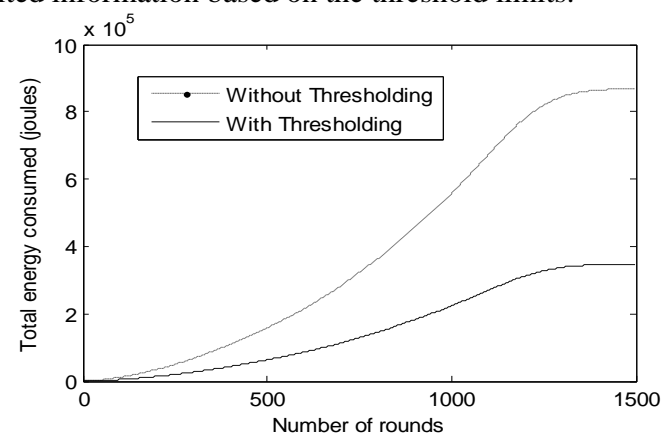

Fig 4 (c) : Total energy consumed by using BBKH

From Fig.4. (c), it can be seen that, as the number of packets sent is less, energy expenditure is too less. Hence, the life of the network has improved.

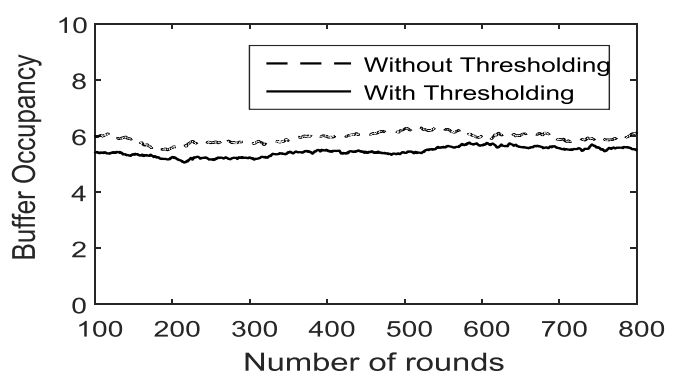

Fig 4 (d): Buffer occupancy by using BBKH 
From Fig4 (d), it is understood that the buffer occupancy is very limited, as only limited data whose value is greater than or equal to the threshold value is obtained from the buffer for transmission. This is the reason for improved energy use and the number of active nodes.

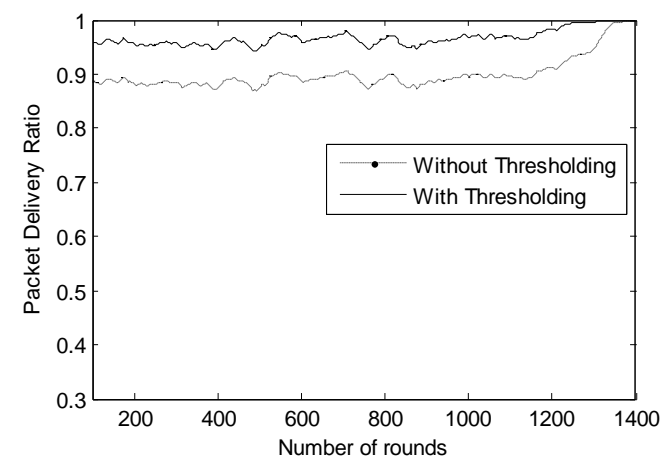

Fig 4 (e) : Packet Delivery ratio by using BBKH

In fig 4(e), the PDR with and without thresholding is compared. PDR is observed to be better with thresholding.

The fig.5 (a) to (e) shows the performance of GA with and without applying threshold concept. It is clear from the fig 5 (a) to (e) that the performance of the GA is less than BBKH.

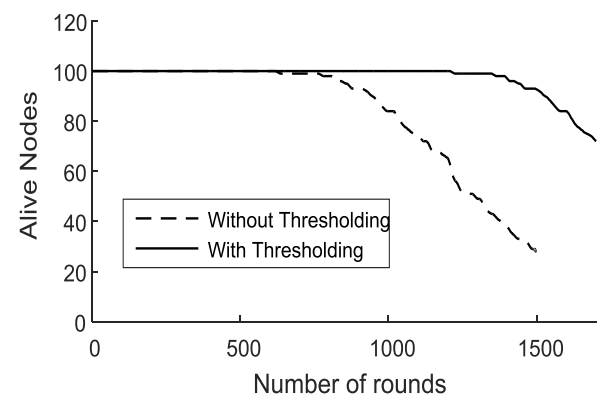

Fig 5 (a) : Total Alive nodes by using GA

As more energy is depleted the number of alive nodes for many rounds is also poor by using GA as revealed by fig 5 (a).

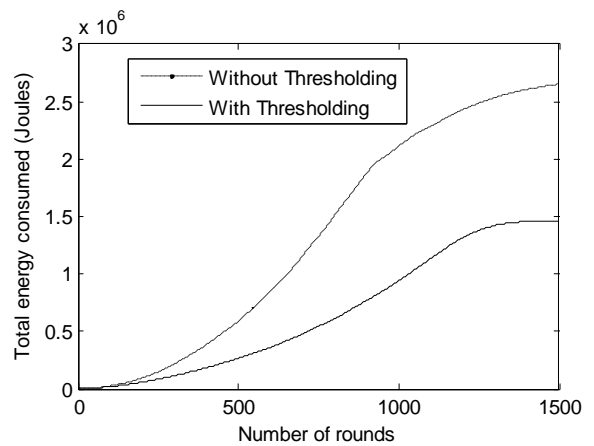

Fig 5 (b): Total energy transmitted by using GA

Before applying, thresholding, almost all the sensed are transmitted to the BS which increases the energy utilization of the nodes as given by fig 5 (b).

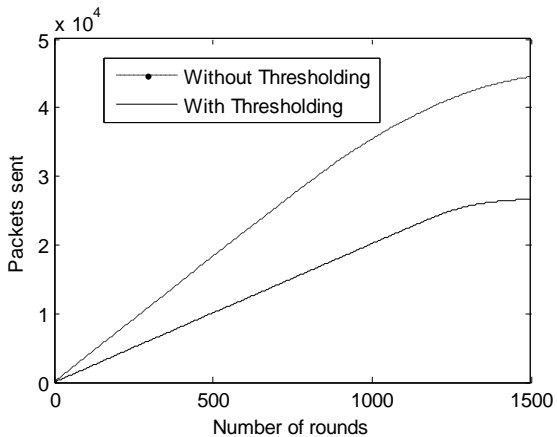

Fig 4 (c): Packets sent by using GA

The total packets sent to $\mathrm{CH}$ is more before thresholding a given by fig. 5 . (c).

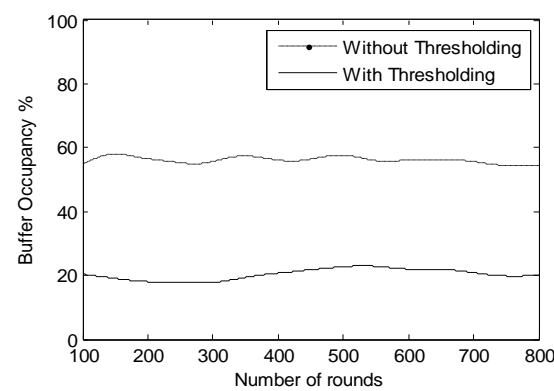

Fig 5 (d): Buffer occupancy by using GA

The buffer occupancy is given in fig 5(d), where the occupancy level is more before applying thresholding concept.

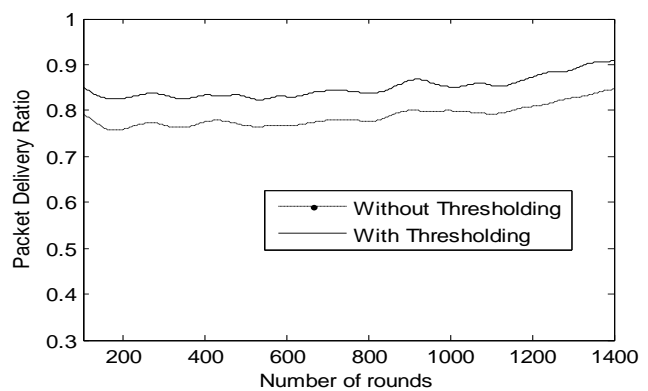

Fig 5 (e): PDR ratio by using GA

Similarly, if all the data sensed was received by the buffer, its occupancy level will be more, leading to a packet drop, which is revealed by the PDR ratio given by fig 5 (e), since the buffer occupancy is more without applying thresholding, more packet drop occurs and shows poor PDR ratio utilization of the SN.

Hence, the proposed BBKH outperforms the classical method GA by applying thresholding concept, which is revealed from the above-given figures.

\section{CONCLUSION}

A threshold based congestion-aware protocol is enforced utilizing the soft computing technique BBKH for network lifetime improvement by optimal $\mathrm{CH}$ selection while considering fair throughput among the flows and lowered buffer occupancy. The obtained solutions are compared with GA. The proposed BBKH improves the network lifetime by $18.80 \%$. The protocol is employed in monitoring the corrosion risk of building constructions. The corrosion risk is discovered at an earlier stage with the help of corrosion 
sensors to avoid further damages. As sensors deployed in newly constructed buildings cannot be reloaded or replaced periodically, the extended life of the sensors is really essential for continuous monitoring of the constructions. The work can be used for applications where the energy utilization of sensors plays a critical part in improving the network lifetime. Chloride ingress is one of the factors which causes cracks in buildings. Chloride sensors can be deployed for monitoring the chloride content. If the chloride ingress crosses the limits, cracks occurrence starts. In future, the work can be extended to monitor the risk of crack occurrence by chloride ingress in concrete structures. The energy efficiency of the sensor nodes can be further improved in the future by developing novel soft computing approaches.

\section{REFERENCES}

[1] Handbook on Repairs and Rehabilitation of RCC Buildings,2002, Director General (Works), Central Public Works Department, Govt of India, Nirman Bhawan.

[2] Wendi Rabiner Heinzelman, Chandrakasan,A and Hari Balakrishnan. 2000 Energy-Efficient Communication Protocol for Wireless Micro-sensor Networks In Proceedings of the $33^{\text {rd }}$ Hawaii International Conference on System Science, Maui, Hawaii.

[3] Manpreet Kaur, Abhilasha Jain and Ashok Kumar Goel 2014 Energy Efficient Two LevelsDistributed Clustering Scheme to Prolong Stability Period of Wireless Sensor Network International Conference on Advances in Computing, Communications, and Informatics (ICACCI).

[4] Sajid Hussain, Obidu Islam and Abdul Wasey Matin 2007 Genetic algorithm for energy efficient clusters in wireless sensor networks In Proceedings of the 4th International Conference on Information Technology, IEEE Computer Society.

[5] Ying Liang, Haibin Yu 2005 PSO-Based Energy Efficient Gathering in Sensor Networks, Springer-Verlag Berlin Heidelberg.

[6] Mohammed Abo-Zahhad, Sabah M. Ahmed, Nabil Sabor and Shigenobu Sasaki 2014 A New Energy-Efficient Adaptive Clustering Protocol Based on Genetic Algorithm for Improving the Lifetime and the Stable Period of Wireless Sensor Networks, International Journal of Energy, Information and Communications Vol.5, Issue 3, pp. 47-72.

[7] Anamika Chauhan, Amit Kaushik 2014 TADEEC: Threshold Sensitive Advanced Distributed Energy Efficient Clustering Routing Protocol for Wireless Sensor Networks, International Journal of Computer Applications (0975-8887), vol 96, no 23.

[8] Arati Manjeshwar and Dharma P. Agrawal 2001 TEEN: A Routing Protocol for Enhanced Efficiency in Wireless Sensor Networks, IEEE.

[9] Fuhr, PL, Spammer, S 1998 Fiber optic sensors in the Waterbury bridge, Proceedings of SPIE: Fourth Pacific Northwest Fiber Optic Sensor Workshop, vol. 3489, pp. 124-9.

[10] James,S \& Tatam,R 2003 Optical fibre long-period grating: characteristic and application, Measurement science and technology 14,pp R49-R61.
[11] Li,H Li,D,S Song,G,B 2004 Recent applications of fiber optic sensors to health monitoring in civil engineering, Engineering Structures 26, pp 1647-1657.

[12] Wolfbeis,O,S (2004) Fiber-optic chemical sensors and biosensors, Analytical Chemistry 76, pp 3269-3284.

[13] Tang,J,L Wang,J,N (2007) Measurement of chloride-ion concentration with long-period grating technology, Smart materials and structures 16,pp 665-672.

[14]Lam,C Mandamparambil,R Sun,T Grattan,K Nanukuttan,S Taylor,S \& Basheer,P 2009 Optical fiber refractive index sensor for chloride ion monitoring, Sensors Journal, IEEE 9 pp 525-532.

[15] Goldmark,A 2011 Report: One in Nine Bridges in America "Structurally Deficient, Potentially Dangerous".

Available:http://www.wnyc.org/story/283331-reportone-in-nine-bridgesin-america-structurally-deficientpotentially-dangerous/

[16] Abbas,Y Olthuis,W \& Van den Berg,A 2013 A chrono potentiometric approach for measuring chloride ion concentration,Sensors and Actuators B: Chemical,vol. 188 , pp.433-439

[17] Hao,S 2009 I-35W Bridge collapse, Journal of Bridge Engineering, vol. 15, pp. 608-614.

[18] Bertolini,L Elsener,B Pedeferri,P Redaelli,E \& Polder,R,B 2013 Corrosion of steel in concrete: prevention, diagnosis, repair, John Wiley \& Sons.

[19] Neville, A 1995 Chloride attack of reinforced concrete: an overview, Materials and Structures, vol. 28, pp. 63 70 .

[20] Ha-Won Song, Velu Saraswathy 2007 Corrosion Monitoring of Reinforced Concrete Structures - A Review, Int. Journal of Electrochemical Science, 2, pp $1-28$.

[21] Siti Fatimah Abdul Rahman, Mohammad Ismail, Norhazilan Md. Noor \& Hazri Bakhtiar 2012 Embedded Capacitor Sensor for monitoring corrosion of reinforcement in concrete, Journal of Engineering Science and Technology, vol. 7. No.2, pp 209-218.

[22] Vti TM Virginia Technologies Inc.www.vatechnologies.com

[23] ASTM C876-91 (1999) Standard test method for halfcell potentials of uncoated reinforcing steel in concrete.

[24] Gandomi,A.H Alavi,A.H 2012 Krill herd: a new bioinspired optimization algorithm, Commun. Nonlinear Sci. Numer. Simulat. 17 (12),pp 4831-4845.

[25] Gaige Wang, Lihong Guo, H. Wang, Hong Duan, Luo Liu \& Jiangh Li. 2012, Incorporating mutation scheme into a krill herd algorithm for global numerical optimization, Neural Comput. Appl., pp 853871.

[26] Wang,G Guo,L Gandomi,A,H 2013 Levy-flight krill herd algorithm, Math. Problem. Eng. Pp 1-14.

[27] Simon,D 2008 Biogeography-based optimization, IEEE Transactions Evol. Computing. 12 (6),pp 702-713. 
[28] Maciej Zawodniok \& Sarangapani Jagannathan 2007 Predictive Congestion Control Protocol for Wireless Sensor Networks, IEEE Transactions On Wireless Communications, Vol. 6, No. 11.

[29] Anamika Chauhan \& Amit Kaushik 2014 TADEEC: Threshold Sensitive Advanced Distributed Energy Efficient Clustering Routing Protocol for Wireless Sensor Networks, International Journal of Computer Applications (0975 - 8887) Volume 96 - No.23.

[30] Arati Manjeshwar \& Dharma P. Agrawal 2001 TEEN: A Routing Protocol for Enhanced Efficiency in Wireless Sensor Networks, IEEE.
[31] Elsener,B Bönhi,H Berke,N,S Chaker,V \& Whiting,D 1990 Corrosion rates of steel in concrete. ASTM STP 1065, Philadelphia, USA, pp. 143.

[32] Broomfield,J,P Langford, A.J.Ewins,A,J Berke,N,S Chaker,V Whiting.D 1990 Corrosion rates of steel in concrete. ASTM STP 1065, Philadelphia, USA, pp. 157.

[33]https://failuremechanisms.wordpress.com/2012/12/01/chl oride-attack/ 